\title{
Criterion for Phase Separation in One-Dimensional Driven Systems
}

\author{
Y. Kafri, ${ }^{1}$ E. Levine, ${ }^{1}$ D. Mukamel, ${ }^{1}$ G. M. Schütz, ${ }^{2}$ and J. Török ${ }^{1}$ \\ ${ }^{1}$ Department of Physics of Complex Systems, Weizmann Institute of Science, Rehovot, Israel 76100 \\ ${ }^{2}$ Institut für Festkörperforschung, Forschungszentrum Jülich, 52425 Jülich, Germany
}

(Received 15 April 2002; published 27 June 2002)

\begin{abstract}
A general criterion for the existence of phase separation in driven density-conserving one-dimensional systems is proposed. It is suggested that phase separation is related to the size dependence of the steadystate currents of domains in the system. A quantitative criterion for the existence of phase separation is conjectured using a correspondence made between driven diffusive models and zero-range processes. The criterion is verified in all cases where analytical results are available, and predictions for other models are provided.
\end{abstract}

DOI: $10.1103 /$ PhysRevLett.89.035702

The existence of phase separation and spontaneous symmetry breaking in low-dimensional systems far from thermal equilibrium has been a subject of recent interest $[1,2]$. While it is well known that these phenomena do not take place in one dimension in thermal equilibrium, several models of driven one-dimensional systems with local dynamics have recently been demonstrated to exhibit both [3-5]. Whether or not a given model exhibits phase separation is in many cases not a simple question to answer, and it may depend on numerical evidence which could be rather subtle.

For example, in a recent three-species model introduced by Arndt et al. [4] (AHR), it has been suggested that one should expect two distinct phase separated states: one in which the three species are fully separated from each other (related to the phase separation observed by Evans et al. [3] in a related model) and the other is a more subtle mixed state whose existence is supported by extensive numerical simulations of systems of finite length and by a meanfield treatment. Subsequently, an analytical analysis of the model has shown that the mixed state is in fact disordered and that in order to see this in simulations one has to study extremely long systems (of the order of $10^{70}$ ), far beyond existing numerical capabilities [6].

In another example introduced by Korniss et al. a twolane extension of a three-species driven system was studied [7]. It has been suggested that while for this model the one-lane system does not exhibit phase separation [8], this phenomenon does exist in the two-lane model. The studies rely on numerical simulations of systems of length up to $10^{4}$. This result is rather surprising and not well understood. It may very well be the case that as for the AHR model, the two-lane model does not actually exhibit phase separation in the thermodynamic limit and that this could be seen only by studying extremely long systems. It would thus be of great importance to find other criteria, which could distinguish between models supporting phase separation from those which do not.

In this Letter we introduce a simple general criterion for the existence of phase separation in density-conserving one-dimensional driven systems. Phase separation is usu-
PACS numbers: $64.75 .+\mathrm{g}, 05.20 .-\mathrm{y}$

ally accompanied by a coarsening process in which small domains of, say, the high density phase coalesce, eventually leading to macroscopic phase separation. This process takes place as domains exchange particles through their currents. When smaller domains exchange particles with the environment with faster rates than larger domains, a coarsening process is expected, which may lead to phase separation. Our criterion quantifies this mechanism and relates the existence of phase separation to the steady-state currents through which domains exchange particles. The criterion is readily applicable even in cases which cannot be decided by direct numerical simulations.

In order to explicitly state the criterion we note that in many models which carry a nonzero current in the thermodynamic limit the current of a finite domain of size $n$ takes the form $J_{n}=J_{\infty}(1+b / n)$ to leading order in $1 / n$. For $b>0$ the current of long domains is smaller than that of short ones, which leads to a tendency of the longer domains to grow at the expense of smaller ones. According to our criterion phase separation takes place at high densities only for $b>2$. Moreover if the current decays to its asymptotic value as $J_{n}=J_{\infty}\left(1+b / n^{\sigma}\right)$, the model is predicted to phase separate at any density for $\sigma<1$, while it is always homogeneous for $\sigma>1$. In some models $J_{\infty}=0$, although, due to lack of detailed balance, the current $J_{n}$ of a finite system is nonvanishing. In this case the system is predicted to phase separate at any density. Models for which $J_{n}$ decays exponentially to zero with $n$ have been analyzed in the past and indeed were shown to exhibit phase separation [3-5].

The results presented above emerge from a careful analysis of a zero-range process (ZRP) which could be viewed as a generic model for domain dynamics in one dimension. To define this process we consider a onedimensional lattice of $M$ sites, or "boxes," with periodic boundary conditions. Particles, or "balls," are distributed among the boxes with the box $i$ occupied by $n_{i}$ balls. The dynamics is defined in the following way: a box $i$ is chosen at random and a particle is removed from it and transferred to a left [right] neighbor with rates $p w_{n_{i}}$ $\left[(1-p) w_{n_{i}}\right]$, where $0 \leq p \leq 1$. The rate $w_{n_{i}}$ depends 
only on the number of balls in that box. The model may be either unbiased $(p=1 / 2)$ or biased $(p \neq 1 / 2)$.

In a grand canonical ensemble, namely an ensemble where the number of boxes $M$ is fixed while the number of balls is allowed to fluctuate with their average number controlled by a fugacity $z$, the steady-state weight of a configuration of the ZRP is known to be [9]

$$
W_{\mathrm{ZRP}}\left(\left\{n_{i}\right\}\right)=\prod_{i=1}^{M} z^{n_{i}} \mathcal{F}_{n_{i}} .
$$

Here $\mathcal{F}_{k}=\prod_{m=1}^{k} 1 / w_{m}$ for $k \geq 1$ and $\mathcal{F}_{0}=1$. In this ensemble boxes are statistically independent with a singlesite occupation distribution function given by $P(k) \sim$ $z^{k} \mathcal{F}_{k}$. Depending on the rates $w_{n}$ the model may or may not exhibit condensation in the thermodynamic limit, whereby the occupation number of one of the boxes becomes macroscopically large. Clearly the rate $w_{n}$ must be a decreasing function of $n$ in order for larger blocks to be favored and to support condensation. It is known [9] that condensation occurs at any density when $w_{n} \rightarrow 0$ with $n \rightarrow \infty$, or when it decreases to a nonvanishing asymptotic value $w_{\infty}$ as $w_{\infty}\left(1+b / n^{\sigma}\right)$ with $\sigma<1$; no phase separation takes place for $\sigma>1$; for $\sigma=1$ phase separation takes place at high densities only for $b>2$.

This model may be used to gain physical insight into the dynamics of driven one-dimensional systems. Occupied boxes represent domains of the high density phase. The currents leaving domains are represented by the rates of the ZRP. This is done by identifying the rate $w_{n}$ associated with a box containing $n$ balls with the currents $J_{n}$ leaving a domain of $n$ particles. A bias in the currents to a certain direction may be incorporated through $p$ as defined above. The existence of a box with a macroscopic occupation in the ZRP corresponds to phase separation in the driven model.

In the following we consider several one-dimensional driven systems and study their domain dynamics by introducing a corresponding zero-range process. By analyzing the ZRP, the existence of phase separation in the original model may be addressed. We begin by considering the AHR model. We show that for this model the corresponding ZRP yields its exact steady-state domain-size distribution. This ZRP does not exhibit phase separation, in agreement with the results of Rajewsky et al. [6]. We then discuss the two-lane model [7] and argue that it, too, does not exhibit phase separation contrary to results of numerical simulations of finite systems.

The AHR model is a three-state model on a ring. Each site is either empty $(0)$ or occupied by a positive $(+)$ or a negative ( - ) particle. The model evolves by a random sequential dynamics in which a pair of nearest neighbor sites is chosen at random and exchanged with the rates:

$$
+0 \stackrel{\alpha}{\rightarrow} 0+; \quad 0-\stackrel{\alpha}{\rightarrow}-0 ; \quad+-\underset{q}{\stackrel{1}{\rightleftarrows}}-+.
$$

This dynamics conserves the number of particles of each type. As in most studies of this model we consider equal densities of positive and negative particles. $\mathrm{Nu}-$ merical simulations suggest that the model has three states [4]: a fully ordered state for $q>1$, in which the system strongly phase separates into its three phases; a mixed state for $q_{c}<q<1$ in which the particles (both positive and negative) in the system condense into a single high density phase separated from a low density gaslike phase; and a disordered state for $q<q_{c}$ where particles and vacancies are homogeneously distributed. On the other hand, exact calculations within the grand canonical ensemble [6] show that the mixed state is in fact disordered, with a finite average length of the high density domains in the thermodynamic limit. Thus the homogeneous and the mixed states constitute a single disordered state. The system therefore exhibits only two states, one fully phase separated for $q>1$ and the other disordered for $q<1$.

It is known $[4,6]$ that for this model the steady-state weight, $W_{L}(C)$, of a given microscopic configuration $C$ is

$$
W_{L}(C)=\operatorname{Tr} \prod_{i=1}^{L}\left[z\left(\delta_{\tau_{i}(+)} D+\delta_{\tau_{i}(-)} E\right)+\delta_{\tau_{i}(0)} A\right] .
$$

Here $L$ is the length of the lattice, $\tau_{j}=+,-, 0$ when site $j$ is occupied by a,+- , or 0 , respectively, and $z$ is the fugacity which controls the average number of particles. The fugacity is the same for positive and negative particles so their average number is equal. The matrices $D, E$, and $A$ satisfy

$$
D E-q E D=D+E \quad \alpha D A=\alpha A E=A
$$

to give the correct steady-state weight. Explicit representations which satisfy this algebra are known $[4,6]$. For our purpose it is sufficient to note that there exists a representation in which $A=|0\rangle\langle 0|$ is a projection operator satisfying $A^{2}=A$, where $|0\rangle$ is a state vector.

We now consider the steady state of this model in an ensemble in which the number of vacancies $M$ is held fixed while the number of particles and thus also the length $L$ of the lattice are allowed to fluctuate. We refer to this ensemble as a grand canonical. A typical configuration of the model consists of blocks of particles bounded by vacancies. By a block we mean an uninterrupted sequence of positive and negative particles, bounded between two zeros. Let $n_{i}(i=1, \ldots, M)$ be the length of the $i$ th block located to the left of the $i$ th vacancy. The block lengths can take the values $n_{i}=0, \ldots, L-M$ and satisfy $\sum_{i} n_{i}=L-M$. The partial trace $W\left(\left\{n_{i}\right\}\right)$ of all weights of microscopic configurations $W_{L}(C)$ consistent with $n_{1}, \ldots, n_{M}$, takes the form

$$
W\left(\left\{n_{i}\right\}\right)=\prod_{i=1}^{M} z^{n_{i}} Z_{n_{i}},
$$

where $Z_{k}=\left\langle 0\left|(D+E)^{k}\right| 0\right\rangle$ is the sum over all weights of microscopic configurations of a block of length $k$. Here we have used the above representation of the matrix $A$. Within the grand canonical ensemble the various domains are statistically independent with a domain size distribution $P(k) \sim z^{k} Z_{k}$. It is known that $Z_{k}$, with the algebra 
(4), is the partition function (sum over all weights) of the partially asymmetric exclusion process (PASEP) on a onedimensional lattice of $k$ sites and open boundary conditions $[10,11]$. The boundary conditions are such that the positive particles are injected at rate $\alpha$ at the left end and are removed from the right end with the same rate. The dynamics in the bulk of the system is given by the same rates as in (2). Moreover, the current in such a system of size $k$ is given by $J_{k}=Z_{k-1} / Z_{k}$ so that $Z_{k}=\prod_{m=1}^{k} 1 / J_{m}$ for $k \geq 1$.

We now turn to define the corresponding ZRP. We consider a ZRP in which each box represents a vacancy in the AHR model. The box $i$ is occupied by $n_{i}$ balls, which corresponds to the number of particles (positive and negative) in the block to the left of the $i$ th vacancy (see Fig. 1). Since in the AHR model the positive and negative currents of a block of size $n$ are equal to $J_{n}$, we choose the hopping rates of the ZRP to be $w_{n}=2 J_{n}$ with a symmetric hopping to the right and to the left. Here again $J_{n}$ is the current of the PASEP in an open system of size $n$. Thus for this ZRP one has $\mathcal{F}_{k}=2^{-k} \boldsymbol{Z}_{k}$. Hence the weight of a given block configuration $n_{1}, \ldots, n_{M}$ in the AHR and the corresponding configuration of the ZRP are the same (up to a $2^{-k}$ prefactor which can be absorbed into the definition of the fugacity $z$ ) [12]. The ZRP provides a simple interpretation of the AHR steady-state dynamics in which blocks interact via exchange of particles. Each block in the AHR behaves as a PASEP with open boundary conditions and equal injection and ejection rates at steady state. Neighboring blocks exchange particles at a rate given by the PASEP current.

We now use the ZRP to study the block size distribution in the AHR model. To do this we use the asymptotic form of the current of the PASEP with $q<1$,

$$
J_{n}=J_{\infty}\left[1+b / n+\mathcal{O}\left(1 / n^{2}\right)\right],
$$

with $J_{\infty}=(1-q) / 4, b=3 / 2$ for $1>q>1-2 \alpha$ and $b=-1$ for $q<1-2 \alpha[10,11]$. Therefore, since $b<2$ in both regimes, no condensate can appear for $q<1$, in agreement with [6]. Moreover, using the form (6) of $J_{n}$ it is easy to show that the block size distribution in the homogeneous phase is given by

$$
P(k) \sim \frac{1}{k^{b}} \exp (-k / \xi) ; \quad \xi=\frac{1}{\left|\ln \left(z / J_{\infty}\right)\right|} .
$$

It is evident that for $b \leq 2$ the average block size diverges

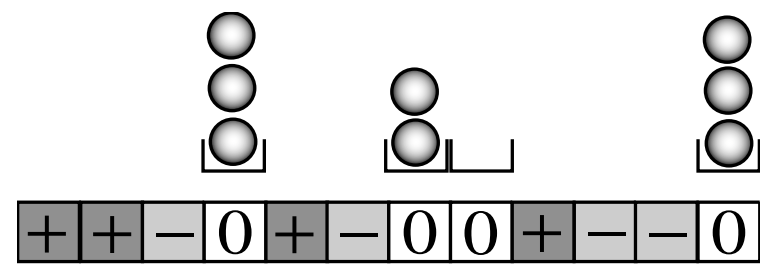

FIG. 1. A typical configuration of the three-state model (bottom) and its corresponding configuration in the ZRP (top). Periodic boundary conditions are imposed on the two models. as $\xi \rightarrow \infty$ and the distribution function is valid for any density no matter how large. No phase transition takes place in this case. However, for $b>2$ the average block size remains finite for $\xi \rightarrow \infty$ necessitating the existence of a phase transition, which results in a macroscopic block at high densities. Note that at $q=1-2 \alpha$ the blocksize distribution function changes in a nonanalytic manner. This point was first noted for the case of $q=0$ in [8].

This result yields interesting insight into the origin of the apparent transition seen in simulations whereby in a certain $q$ interval the correlation length $\xi$ becomes exceedingly large. It can be shown [13] that the $q$ dependence of the correlation length $\xi$ is introduced by the higher order corrections (for example, $c / n^{2}$ ) to the current (6). We have calculated the correlation length $\xi$ of a ZRP with rates $w_{n}=1+3 / 2 n+c / n^{2}$ for a given density as a function of the parameter $c$ (corresponding to changing q) [13]. We find that $\xi$ exhibits a sharp increase of a few orders of magnitude over a narrow range of values of $c$. This reflects itself in large (but finite) blocks and an apparent phase separation in direct simulations.

The physical picture emerging from this analysis offers a rather robust mechanism for phase separation, and we conjecture that it has a more general validity. We expect it to apply to other conserving driven models even though an exact correspondence to the ZRP may not be evident.

We now demonstrate the use of the conjecture for the two-lane model introduced by Korniss et al. [7]. This model is a generalization of (2) with $q=0$ to two lanes. Here in addition to the hopping process (2) within each lane, particles may hop to neighboring empty sites on the other lane with rate $\gamma \alpha$, and to exchange with a neighboring particle on the other lane with rate $\gamma$. Numerical studies of the model have suggested that for large enough $\alpha$ the system phase separates [7]. In this state the particles condense into a single high density block. However, physical insight into the phenomenon is lacking. In particular, it is not understood why the two-lane model seems to exhibit phase separation while its singled-lane version does not [8]. In order to apply the conjecture, the current $J_{n}$ of a block of size $n$ is calculated for the two-lane model. This is done by considering an open two-lane system of length $n$ with no vacancies where particles are injected and ejected at the boundaries with equal rates $\alpha$. Setting $\gamma=1$ as in [7] we show the results of numerical simulations for several values of $\alpha$ in Fig. 2. By comparing the results for the corrections to the current $\Delta_{n}=\left(J_{n}-J_{\infty}\right) / J_{\infty}$ with the line $2 / n$ it is easy to see that in this case $b<2$. In fact, the curve may be best fitted to $b \simeq 0.8$. Our conjecture thus implies that phase separation does not take place in this model. Note that even if one tries to fit $\Delta_{n}$ to $1 / n^{\sigma}$ with $\sigma \neq 1$ one finds that $\sigma \gtrsim 1$ yielding the same conclusion. We note that simulating the open systems and obtaining the asymptotic behavior of the current involves a relatively modest numerical effort, as one needs only to simulate rather small systems. This should be compared with the huge systems which are needed in order to demonstrate 


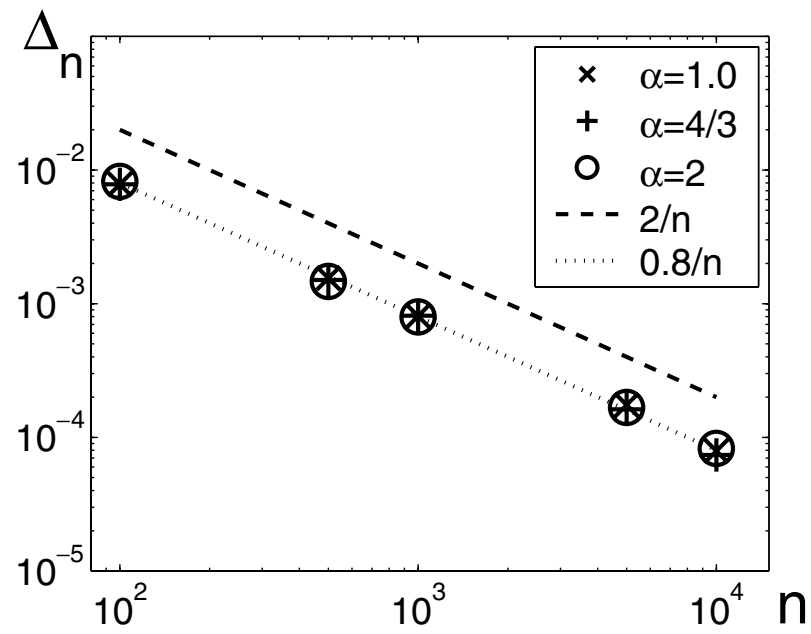

FIG. 2. The finite-size corrections to the current $\Delta_{n}=\left(J_{n}-\right.$ $\left.J_{\infty}\right) / J_{\infty}$ in the two-lane model with open boundary conditions, for different system sizes. Here $\gamma=1$.

the lack of phase separation in direct simulations. Indeed, the conjecture suggests that the apparent phase separation found in numerical studies is due to simulations of systems much smaller than the typical domain size.

We have studied another extension of model (2), whereby the hopping rates depend on both nearest and next-nearest neighbors. In these models we have shown that $b=3 / 2$ with $\sigma=1$. The conjecture implies no phase separation, in contrast to our direct simulations.

We now apply the conjecture to a class of models with vanishing $J_{\infty}$. To this end, consider model (2) with $q>1$. As before, we define a block as a sequence of particles, both positive and negative, bounded by two vacancies. The corresponding open system is the PASEP with $q>1$, with open boundary conditions and equal injection and ejection rates, studied in [11]. The current in such a block, as in the corresponding open system, is exponentially decreasing with the block size, $J_{n} \sim q^{-n / 2}$. This is easily understood, as the particles are moving against the bias when $q>1$. Using the conjecture the system is expected to phase separate for any density. Several similar models with exponentially decaying currents have been shown to exhibit a strongly phase separated state [3-5].

We end by reiterating the assumptions involved in the conjecture, which relates phase separation to the currents $J_{n}$ of finite blocks. It is assumed that the current flowing through a block is given by its steady-state value and is independent of its neighboring blocks. This may be justified by the fact that the coarsening time of large domains is very long, and the domains have a chance to equilibrate long before they coarsen.

Although the criterion introduced in this Letter has not been proved to hold in general, its underlying physical mechanism for phase separation is rather robust, suggesting a broad applicability. It is of interest to test the conjecture on other models to check its general validity. In the models exhibiting phase separation we have studied so far, the current $J_{\infty}$ vanishes. It would be interesting to find a one-dimensional driven model for which $J_{\infty}>0$ and $b>2$. According to the conjecture such a model should exhibit a novel type of phase separation.

We thank M. R. Evans for many discussions and suggestions and $\mathrm{C}$. Godrèche for very fruitful discussions on the AHR model. He and S. Sandow are thanked for communicating to us a preliminary version of [8]. We also thank I. Kanter and V. Rittenberg for discussions. The support of the Israeli Science Foundation is gratefully acknowledged. G. M. S. thanks the Einstein Center and Deutsche Forschungsgemeinschaft for support.

[1] M. R. Evans, D. P. Foster, C. Godrèche, and D. Mukamel, Phys. Rev. Lett. 74, 208 (1995).

[2] For a recent review, see D. Mukamel, in Soft and Fragile Matter: Nonequilibrium Dynamics, Metastability and Flow, edited by M. E. Cates and M. R. Evans (Institute of Physics Publishing, Bristol, 2000); cond-mat/0003424.

[3] M. R. Evans, Y. Kafri, H. M. Koduvely, and D. Mukamel, Phys. Rev. Lett. 80, 425 (1998); Phys. Rev. E 58, 2764 (1998).

[4] P. F. Arndt, T. Heinzel, and V. Rittenberg, J. Phys. A 31, L45 (1998); J. Stat. Phys. 97, 1 (1999).

[5] R. Lahiri and S. Ramaswamy, Phys. Rev. Lett. 79, 1150 (1997); R. Lahiri, M. Barma, and S. Ramaswamy, Phys. Rev. E 61, 1648 (2000).

[6] N. Rajewsky, T. Sasamoto, and E. R. Speer, Physica (Amsterdam) 279A, 123 (2000); T. Sasamoto and D. Zagier, J. Phys. A 34, 5033 (2001).

[7] G. Korniss, B. Schmittmann, and R. K. P. Zia, Europhys. Lett. 45, 431 (1999); J. T. Mettetal, B. Schmittman, and R. K. P. Zia, cond-mat/0110301.

[8] C. Godrèche and S. Sandow (unpublished).

[9] See, e.g., F. Spitzer, Adv. Math. 5, 246 (1970); M. R. Evans, Braz. J. Phys. 30, 42 (2000); O. J. O'Loan, M. R. Evans, and M. E. Cates, Phys. Rev. E 58, 1404 (1998).

[10] T. Sasamoto, J. Phys. A 32, 7109 (1999).

[11] R. A. Blythe, M. R. Evans, F. Colaiori, and F. H. L. Essler, J. Phys. A 33, 2313 (2000).

[12] Note that each block configuration in the AHR model carried an extra degeneracy which is bounded from above by $L$ as compared to the corresponding ZRP. This degeneracy corresponds to the number of ways of placing a given block configuration on a lattice of length $L$. It does not affect the results in the thermodynamic limit.

[13] Y. Kafri, E. Levine, D. Mukamel, and J. Török, condmat/0206145. 